\title{
New Management Methods for Feature and Preference Interactions
}

\author{
Zohair Chentouf, Soumaya Cherkaoui, and Ahmed Khoumsi \\ Department of Electrical and Computer Engineering \\ Université de Sherbrooke \\ 2500, boulevard Université \\ Sherbrooke (Québec) J1K 2R1 CANADA \\ zohair.chentouf@hermes.usherb.ca \\ \{soumaya.cherkaoui, ahmed.khoumsi\}@usherbrooke.ca
}

\begin{abstract}
This paper deals with a complicated Feature Interactions (FI) problem that results from new emerging telecommunications architectures, IP telephony for example. That is because these architectures introduce new complications since service providers and end users have the possibility to express their preferences in more sophisticated ways and non telephony applications are combined with telephony services. The article provides examples of new FI that have not been reported before. The main contribution of this work is that it proposes new FI management methods that are required to solve the new FI complications. Old management methods are also considered and some of them are enhanced in order to adapt to the new problem context. Intelligence requirements necessary to implement the proposed management model are finally underlined.
\end{abstract}

\section{Introduction}

The Feature Interactions (FI) problem can be defined as the situation in which two feature instances undesirably affect one another when running together $[1,2]$. In the literature, this definition or similarly formulated ones are adopted [3]. They have in common to associate undesirability with altered feature behavior or user confusion with that behavior. A scholastic example usually evoked is the FI that occurs between Call Forwarding (CF) and Originating Call Screening (OCS) services. Suppose user A has OCS with which he has specified $\mathrm{C}$ as forbidden destination address. Thus, calls initiated from A's device to C are blocked by OCS. Suppose user B has CF with which she has specified $\mathrm{C}$ as forwarding-to address. This means that every call incoming to B's address is automatically redirected to C. Assume that user A calls user B. A's OCS will not block the call because the destination address is not C, which is the forbidden one. But B's CFU will redirect the call to C. Consequently, user A will find himself talking to $\mathrm{C}$ despite he has OCS that blocks calls to $\mathrm{C}$.

In order to manage FI, three main operations may be used: avoidance, detection and resolution. Avoidance means to intervene at service, protocol and/or architecture design stages in order to avoid interactions whose causes are situated at some of these levels, respectively. Detection aims at detecting interactions using suitable test 
methods, performed on service models which are usually obtained using a suitable service modeling language. Resolution means to find and apply a solution to already detected FI. This article interest goes to FI detection and resolution. So, when the term "management" is used, it will mean these two operations.

FI management can be performed at two stages:

- off-line: before services are deployed in the network, or

- on-line: either when services are not yet publicly available (test phase) or after the services are available to public. [3]

We should also distinguish between two situations into the phase where services are available:

- management is done at runtime, i.e. when services are running, or

- it is done before runtime, when services are not running.

New emergent telecommunications architectures such as IP telephony, give more possibilities for end users to express their preferences and personalize services. They also bring the possibility to have services running in end user devices and third party provider servers. As user preference modeling possibilities grow, the interactions between preferences and between preferences and services also become more and more frequent and complex.

In [5], we have specified an architecture for runtime FI detection in IP telephony based on SIP protocol [6]. In the present article, the aim is at completing that specification by adapting it to the FI problem new constraints and management requirements without being restricted to IP telephony. The reached generality level permits to apply the model to the other emergent architectures such as JAIN [7] and 3GPP [8]. We propose new runtime and before-runtime management methods that should be added to old ones. Such a management, which includes runtime and beforeruntime methods, is called hybrid. The new methods and old ones are consistently gathered into a single model. We here present a general model that encompasses many implementation options. This will be explained in the following sections.

In [4], we propose to formulate user preferences using a preference rule formalism. A preference rule is a condition-action rule, where condition part encompasses the events that, if they occur, the user preference, represented by the action part, is executed. Preference rules should be written in a suitable user preference language. At runtime, the detection procedure execution environment should be able to extract information from the exchanged call messages. For example, the caller and callee addresses, the communication subject, the used media type, etc. Such information is necessary for FI detection and should be modeled using the same language as services and preference rules.

It is worthy noting that, depending on the telecommunications architecture, the preference language expressive power and the deployed services, one can have preference rules that behave like services, service behaviors that can not be modeled as preferences and preference rules behaviors that are not available as services. For example, in a SIP architecture where preferences are modeled using CPL [9], forwarding all incoming calls to a given address can be modeled as a preference rule. If the Call Forwarding service is available, the same behavior may be available as a service provided by the service vendor and as preference rules programmed by end users. Otherwise, the only way to have this behavior is to model it as a preference 
rule. Call Intrusion, which means the possibility to interrupt a call between two users in order to talk to one of them, is not possible to model as a preference rule because CPL does not permit to model such a behavior.

The paper is organized as follows. Section 2 provides a taxonomy for the FI problem causes and presents a list of examples of new FI that are engendered by the characteristics of emergent telecommunications architectures. Section 3 describes the architecture model we adopt and the proposed associated FI management model. It shows, case by case, how FI presented in Section 2 should be managed by this model. The paper ends with some concluding remarks in Section 4.

\section{Taxonomy of FI Causes}

The main characteristics of new emerging telecommunications architectures are the following:

- Non telephony applications, such as email, can be involved with telephony ones.

- Some services, preference rules and non telephony applications are roomed by end user devices.

- End users specify preference rules, but they do not expect to have to detect and solve FI.

These characteristics translate in a heterogeneous architecture that encompasses nodes with different technologies. For the sake of generality, let us suppose the following hypothetical architecture model, which has those characteristics:

- End users can have preference rules on their devices and in a network device as well.

- They also can have services in their devices and in the network.

- They also can have non telephony applications that are combined to services and preference rules, in their devices and in the network.

In such an architecture, that contains characteristics of emerging telecommunications ones, the following FI causes may arise:

- Violated assumption

Services and preferences are modeled supposing a set of assumptions about the system behavior. The latter encompasses basic architectural policies about addressing (one-line-per-user or many-lines-per-user, 911 is the emergency address), under which conditions signaling events are issued ("busy" is issued if the callee line is in use), billing, etc. In some cases, a service or preference rule behavior alters an assumption or hides some of its results from other services or preference rules. Thus, the latter can not react as expected despite the call processing context is really the one they should react to.

Example 2.1: user B has Call Waiting service and user A a preference rule that sends an email to the callee if the latter is busy. Suppose B's Call Waiting implementation does not send a "busy" response because, for example, the caller is connected to a music on hold server that sends call acceptance response. If user A hangs on because he does not want to wait, the preference rule envisioned by user A will not be run despite user B is busy. 
- Incompatible actions

There are four interaction causes that belong to this category:

- One action forbids the other one

Example 2.2: user A has a preference rule that forbids forwarding calls initiated by A and user B has Call Forwarding service. If A calls B, there will be an interaction between two actions: the preference rule action that forbids forwarding calls and the Call Forwarding service action that forwards calls.

- The two actions cause user confusion

Example 2.3: user B has a preference rule that forwards to her mobile all incoming calls from persons with whom she had a long distance call since last Monday. On Wednesday, B specifies A as a forbidden caller in Terminating Call Screening service. Suppose A calls B. Depending on the service and preference rule implementation and network conditions, they may be run together. This would cause a confusion to user A since he will be forwarded to B's mobile and receive a message like "Sorry, call later" from the screening service.

- It is impossible to run the two actions together

Example 2.4: in the precedent example, depending on the implementation and network conditions, it may be impossible to run the preference rule and the service together and one among them may be excluded. This may result in violating B's intention.

- The attempt to run the two actions together causes a system ambiguity

Example 2.5: in the precedent example, if the service and preference rule are located on the same node, it may be ambiguous for that node to run one or the other. This depends on their implementation and the rooming node policies. It was not reported for these service and preference rule to engender an ambiguity, but the situation is known to occur in the Intelligent Network when, for example, Call Waiting and Call Forwarding services are triggered by the same event $[1,10]$.

- Forbidden communication content

The communication content relates to the conversation subject or the media type and/or content that is conveyed by the exchanged messages.

Example 2.6: suppose user A has a preference rule that forbids to receive web pages that contain advertisement of religious sects. User B, being enthusiastic member of a sectarian organization, she has a preference rule that sends to all callers the organization's web page. This situation results in a preference conflict if A calls B.

- Ambiguous event content

Some signaling events may be associated a media in order to produce a user notification effect, such as busy tone. The media is the event content. The interaction occurs when the user associates the same content to two distinct events.

Example 2.7: the same tone or melody associated to incoming call and email reception events.

- Action priority order conflict

Depending on the architecture, a negotiation mechanism may be available such that services or preference rules of the caller and callee exchange a set of action proposals and counterproposals in order to negotiate a common solution which results in a single action to perform. The proposed actions should be weighted in order to express the preferable priority order. The other side issues a counterproposal by suggesting its own weighting order for those actions. If the proposal and counterproposal contain different weighting orders, there is an action priority order conflict. The negotiation 
mechanism should permit the two sides to negotiate a common solution by relaxing their preferences.

Example 2.8: user B has a preference rule that is triggered on call request reception. This rule specifies a list of forwarding destinations in the following preferable order: secretary (high), voicemail (medium), mobile (low). This proposal is communicated to the caller, say user A. The latter, however, has specified the following preferable order: secretary (medium), voicemail (low), mobile (high). This list is sent to user B as a counterproposal. Since it is different from B's proposal, there is an action priority order conflict. The two devices should then negotiate a common action to perform. Notice that the negotiation protocol may be different from the one adopted in this example. Weights may be expressed differently.

\section{- Timing}

Timing means at what time a particular event occurs or for how long an event lasts. Events may be delayed by external sources, such as the user, and then may have different effects. It is known that in POTS, for example, how long the switchhook is pushed can determine whether it signifies a flashhook or a disconnection [1]. The following example is a new FI that has not been reported before and that may occur in new telecommunications architectures.

Example 2.9: user B has a user device-located Forward-On-No-Answer service for which she has specified a preference rule that triggers this service after 6 rings, when a call request is received. User $A$ has a user device-located Email-On-No-Answer service for which he has specified a preference rule that triggers it after 4 rings. So, this service sends an email to the user called by A if this user does not answer the call after 4 rings. Suppose A calls B when she is not available. After 4 rings an email is sent to $\mathrm{B}$ and $\mathrm{A}$ will no more hear ringing tone and then will hang on. Here is an interaction between the two preference rules because intentions of both users are not respected: A's one because his device concludes too early that B is not available, and B's one because her device does not actualize her intention at right time (to forward the caller). If user A specified a number of rings equal or greater than the number specified by user B, he would be forwarded to another person and this could be preferred for both users.

- Too generalized action condition

When specifying preferences, end users could neglect to consider special cases to be treated conveniently. In such a case, they associate a too generalized condition to a given action.

Example 2.10: user B has Do-Not-Disturb service programmed to reject all incoming calls on a specified interval of time, say between 10.30 and 13.00. Suppose user A calls user B on that interval of time, specifying a high call priority order and a precise subject. The call request will be rejected by B's Do-Not-Disturb service. There is an interaction between A's preference that translates in assigning a high call priority order and B's Do-Not-Disturb service.

\section{- Preference formulation incompleteness}

When specifying preferences, end users could neglect to consider all the possible cases. Thus, the preference formulation results in neglecting some cases that should be reacted to by the same action or an equivalent one. 
Example 2.11: user A does not want to communicate with one of his old clients, say user B, because the latter became a competitor. A specifies B's address as a forbidden caller address in Originating Call Screening service and a forbidden callee address in Terminating Call Screening service. Therefore, he is expecting no call will be originated towards B and no call from B will be answered. Suppose user A's secretary calls B for some reason. The call attempt does not succeed because it is intercepted by Originating Call Screening, but an email is sent to B because A has a preference rule that sends an email to potential clients, on every call attempt, may they answer or not, with an updated new product list. Consequently, there is an interaction between Originating Call Screening service and the preference rule, which results in violating user A's intention. The latter being not to communicate with B.

Notice that the following FI causes are known in the FI problem [1]: violated assumption, incompatible actions, ambiguous event content, and timing. The following are new FI causes: forbidden communication content, action priority order conflict, too generalized action condition, and preference formulation incompleteness. Examples 2.3-2.7 and 2.9-2.11 are new interactions. They involve user preferences. Example 2.1 involves user preference but a similar one, that involves services only, is presented in [1]. Example 2.2 is quoted in $[2,11]$.

\section{FI Management Methods}

\subsection{Related Work}

\section{Detection}

Most FI detection work reported in the literature uses formal methods. Services are modeled using a formal language. Then, formal techniques are used in order to detect possible interactions. The detection is done off-line or on-line but before runtime. The commonly used formal techniques are temporal logic [12], theorem proving [13], Petri nets [14], extended finite state automata (SDL language for example) [15], processes algebra (LOTOS language for example) [16], etc. Informal methods are also used to detect FI off-line. For example, in [17] natural language processing is used to identify interactions between service logic requirements modeled by textual descriptions.

\section{Resolution}

FI resolution uses two main methods: restriction and negotiation. Restriction results in specifying a precedence or exclusion rule to apply in order to avoid to two services to interact. Precedence means to run one service before another and exclusion means to exclude one service and run only the other one.

The solution proposed in [18] uses software static and mobile agents to apply restriction rules specified during an off-line detection and resolution phase. Other examples of restriction can be found in [19-21].

There are mainly two models that use negotiation: negotiating agents and polite service. 


\section{- Negotiating agent model}

The negotiation method to solve FI was inaugurated by the work due to Griffeth and Velthuijsen [11]. These authors proposed and prototyped a negotiation architecture in which end users have the possibility to specify their preferences. The negotiating agents represent users, network operator and network devices. FI are detected and solved at runtime. The resolution is performed in respect of user preferences. The key idea of the model is that resolution should not favor one feature over another, using restriction. When an agent intends to perform an action, it proposes that action to other agents with whom it is involved in the current call. The latter agents may refuse the proposed action because it violates their preferences. In such a case, each agent proposes another action or set of sub-actions to be substituted to the first one.

In the architecture proposed by Amer et al [2], network and user devices are controlled by device agents. User preferences are contained in user agents. A device agent may contain multiple feature agents that implement features. Users specify their preferences in terms of fuzzy values they assign to preference parameters. Those values express the percentage of preference the user assigns to each parameter.

- Polite service model

In this model, management also operates at runtime but does not use a detection phase before resolution one. It is proposed to operate in an IP telephony architecture based on SIP protocol [6]. Agents represent user devices. The key idea underlying [22] is that when an agent intends to perform an action that may affect another agent involved in the same call, it should ask that agent if it agrees or not. For example, Call Forwarding should not be executed before asking the authorization of the caller's agent. If the latter refuses to be forwarded, the callee agent should propose another action, voicemail service for example. The other work that has to be classified under polite service model is [23]. SIP is extended in order to convey negotiation communication. The latter, as in [22], aims at avoiding actions that may engender interactions. The call request conveys a list of action alternatives the caller agent proposes to be performed by the callee agent. The callee agent either accepts one action or refuses the whole list. This translates in accepting or rejecting the call, respectively.

\subsection{New Management Methods}

In order to manage FI, the architecture model that has been adopted in the beginning of Section 2 needs to satisfy the following requirement: end user devices, non telephony servers and any telephony node that may be involved in FI should contribute together in order to manage FI.

For this requirement to be satisfied, we suppose the architecture enhanced by FI management (FIM) agents located in all the devices and components that should cooperate in managing FI. Lets us call them like follows:

- UFIMA: User device Feature Interaction Management Agent, located on user devices.

- NFIMA: Network component Feature Interaction Management Agent, located in the telephony and non telephony nodes.

- CFIMA: Central Feature Interaction Management Agent, located in the network. 
We suppose UFIMA and CFIMA capable of detecting FI. They also should contain a FIM knowledge that permits them solving the FI they detect. All the agents should be able to communicate with each other. Services and preference rules are coded in a common language. We suppose UFIMA has models of services and preference rules that are in the corresponding user device and in the network as well. CFIMA is supposed to be periodically provided by UFIMA and NFIMA with models of services and preference rules located in user and network devices. The FIM designer should determine which FI to be detected by UFIMA and which by CFIMA, in order to avoid redundancy in management effort. For example, UFIMA may be designed to deal with preference incompleteness FI only. We propose that FI detection should be done before runtime for the services and preference rules that belong to the same user. We propose also that it should be done before runtime, for services and preference rules that belong to users which frequently call each other. At runtime, detection is done for users which are not frequent callers to each other and for services and preference rules that contain information that is specified at runtime only, like in Example 2.10 as explained below.

Some FI causes that arise in the architectural new context we have adopted need new management methods:

- Preference formulation completion

There are two kinds of preference completion:

\section{Preference generalization:}

The user preferences are formulated in a way that covers only a part of the situations they should deal with and neglect others. The preference formulation should then be generalized to the remaining situations. This is done before runtime by UFIMA or CFIMA, depending on whether the preference rule is in the user device or in the network, respectively. Preference generalization method deals with FI whose cause is preference formulation incompleteness but may also solve FI whose cause is violated assumption. The preference generalization FI resolution method is a new one that has not been reported in the literature.

Example 2.1 FI belongs to violated assumption type and may be managed in the following manner. Before runtime, user A UFIMA detects that "busy" event that is specified in the preference rule as triggering condition may be deduced from other events too, like "put on hold". This is a preference formulation incompleteness. So, depending on its FIM knowledge, UFIMA either:

- warns user A giving him a list of other situations that may be considered as implying "busy" event, and/or

- generalizes user A's preference formulation by adding those situations in the triggering condition. Depending on UFIMA knowledge, the update of the preference rule triggering condition may add as another condition, the fact that the user hangs on, in order to avoid having the situation where the user waits for the call to be answered and sends an email that means he will not talk.

In Example 2.11, the FI belongs to another cause: preference formulation incompleteness. It occurs because user A's screening preference expressed by the screening services logic is incomplete. Screening should be done on calls but also generalized to emails. For this kind of FI to be solved, preference generalization should be applied before runtime. UFIMA detects the preference incompleteness and then, depending on the FIM knowledge, either: 
- warns the end user and may help him expressing more complete preference rule, or

- directly performs the necessary completion without help from the end user. In both example cases, whether UFIMA asks the user or acts directly, is to be decided by the FIM designer when implementing the preference generalization method. The designer has also to determine when to perform the detection, for example, each time a service or a preference rule is updated.

\section{- Exception}

The user preferences are formulated in a way that does not take into consideration some special situation cases, where the user preferences should not be applied or should be modified. The exception resolution method prevents user preferences to be applied to those special cases, may be after asking the user. It may also modify user preferences in order to avoid FI. Whether asking the user or not, is an implementation option that may depend on the nature of involved services or preference rules. Notice that exception is not a new management method but it needs to be adapted to FI. In fact, the negotiation performed in $[2,11]$ aims at performing an exception in order to relax a too generalized preference.

In Example 2.9, the FI is caused by a timing situation. It may be solved by an exception. The example formulation supposes the two services are located on user devices. Assuming users A and B frequently call each other, CFIMA detects before runtime the FI and informs corresponding UFIMAs about it. The latter should ask users in order to perform an exception by changing the specified number of rings. If users $\mathrm{A}$ and $\mathrm{B}$ are not potential callers to each other, CFIMA detects the FI at runtime. If UFIMA are designed to negotiate such an interaction type, CFIMA inform corresponding UFIMA in order to negotiate. If they can not negotiate, CFIMA may be designed to let processing the call and then inform UFIMA about the FI. UFIMAs then behave like in before-runtime situation.

The FI caused by the too generalized action in Example 2.10 can be solved by performing an exception at runtime. It is not possible to perform the resolution before runtime because the call subject and emergency level are specified at runtime. UFIMA should be able to check at runtime if the priority order specified in caller request (user A in this example) would interest user B. UFIMA should be able to learn and maintain a keyword repository of the subjects preferred by the end user. When receiving a call with pretended high emergency, UFIMA then uses that repository to decide if it should warn the end user despite he has blocked incoming calls.

\section{- Device cooperation}

In order to avoid the FI of Example 2.6, cooperation is needed from web servers. So, the FIM becomes a responsibility shared, not only by telephony devices, but also by all other components which room applications that are combined with telephony services. This responsibility is carried by NFIMAs. A web content screening feature should be available on the involved web server. Before runtime, user A UFIMA informs the suitable web server NFIMA and the latter instructs the screening feature to screen incoming sectarian web content. In the same manner, each involved non telephony device should room a NFIMA in order to manage FI. In order not to be a burden for the device, NFIMA should operate autonomously in order to cooperate with other FIM agents. 


\section{- Negotiation}

Action priority order conflict needs a negotiation protocol that is different from the ones reported in the related work for FI problem (see Section 3.1). The resolution of Example 2.8 FI should be done at runtime in the following manner. When user B's preference rule is triggered, the list of weighted actions is communicated to user A UFIMA. The latter issues a counterproposal in which it specifies other weight values for those actions. Then, user B UFIMA proposes other values in an attempt to adapt to user A UFIMA counterproposal. The process may run over many steps until reaching a common solution that is to agree on a unique preferred action to perform. If the negotiation does not result in a common solution in a reasonable time, CFIMA should intervene to apply a solution it chooses from the previously rejected proposals or to apply a restriction. This depends on the FIM knowledge it possesses.

\subsection{Applicability of Old Management Methods}

The management models presented in Section 3.1 do not cater with all the constraints considered in this article and which better reflect the new telecommunications reality characterized by network heterogeneity and end user programmability (preference formulation advanced possibilities). The proof is that the management model we propose introduced two new FI management methods: preference generalization and device cooperation; and reformulated two old ones: exception and negotiation.

Formal methods do generally not convene to the architecture model we adopted because end users would be constrained to interpret formal results. They have not the required technical skills to do that. Formal methods also can not be used in the situation where management should be done at runtime. The other management models have the common shortcoming to load services before detecting if they will cause interactions, except [18]. If the resolution needs to exclude a service, this will not be possible since it already started to run, and if a negotiation should be performed, it will be constrained by the actions already performed by the loaded services. Another work [18], presents a different approach from the one adopted in the present work since it partially relies on code mobility. The model of Griffeth and Velthuijsen supposes a hierarchy of the possible actions to perform by services such that, if an action causes an interaction, it may be replaced by another action or set of actions. This assumption does not apply to all the possible actions however. Polite service model represents a partial solution. In fact, it deals only with services and preferences that are triggered by the action to issue or receive a call request but not other SIP messages and events. It also considers only services that involve multiple users and not conflicting services that belong to a single user. Finally, the interaction causes considered in negotiating agents and polite service models do not cover the list presented in this article, particularly the new FI causes. The FI management model presented in this article avoided these shortcomings in order to satisfy the new FI requirements.

Nevertheless, old FI management methods are still applicable to the examples that have not been treated in Section 3.2.

- Example 2.2: at runtime, when A calls B, CFIMA detects an incompatibility between B's Call Forwarding service and A's preference rule that forbids 
forwarding calls initiated by A. CFIMA has no solution other than applying a restriction that excludes B's Call Forwarding and rejects the call request.

- Example 2.3: if user B's preference rule and Terminating Call Screening service are located on her device, UFIMA performs a FI detection and detects two incompatible actions. Depending on UFIMA FIM knowledge, it either:

- warns user B about the incompatibility or

- decides to apply a restriction at runtime that privileges screening and excludes forwarding, for example. If this is done, it should be sure that it does not violate B's intentions. This is expected to be guarantied by the fact that UFIMA policies do not violate the user intention.

If both or one among the preference rule and the service are located in the network, and depending on the implementation management solution policies, CFIMA may do that detection and then behave like UFIMA does in the previous case.

- Example 2.7: The two events are given the same melody by the user device. Before runtime, UFIMA performs a FI detection and detects a content ambiguity. It then warns the user about the ambiguity.

\subsection{New Intelligence Requirements}

The FI management examples presented in Section 3.2 have shown that more intelligence is required to manage FI, especially when this is done by the new methods introduced by the present article. In fact, preference completion (generalization and exception) need from UFIMA to communicate with the user, explain him the FI situation and assist him in completing his preference formulation (examples 2.1 and 2.11). These are new capabilities needed to cater with the growing complexity of the new FI problem. Preference completion (generalization and exception) also requires from UFIMA to be pro-active. Pro-activity means the capacity of getting decisions on the right moment without control from another entity [24]. Preference completion also requires, in some cases, UFIMA to be capable of learning user preferences (Example 2.10). Some FI resolution cases require CFIMA to inform UFIMA about the FI in order to solve it (Example 2.9). This delegation operation is a special device cooperation way. In order to guarantee cooperation from non telephony nodes, they should have a NFIMA. The latter should act autonomously and should be able to control some operations of the hosting node, for example to screen web pages (Example 2.6). The negotiation protocol proposed in the present article is different from the one used in the negotiating agents and polite service models (see Section 3.1). In fact, this protocol is a generalization of those of these two models. Recall that the protocol used in the negotiating agents model proposed by Amer et al consists in one proposed action to which is opposed one action counterproposal. The one used in polite service model permits to choose one action from a proposed list. These two protocols are special cases of the one we proposed for Example 2.8. The protocol, however, needs more intelligence in the negotiating UFIMAs. UFIMA should have suitable knowledge and policies to relax preferences depending on the caller preferences. Notice also that, at our knowledge, negotiation before runtime our model introduced, is a new management method since, in the literature, negotiation is done at runtime. 


\section{Conclusion}

This article presented the complicated FI problem that arises from considering the interactions that involve user preferences and non telephony applications with services. The presented examples of new interactions prove this concept. The new formulation of the problem implies new management methods that, as it has been shown with examples, solve the new complications brought by the growing user preference formulation possibilities and the combination possibilities of services with non telephony applications. The new management methods, however, require more intelligence capabilities in user and network devices. It is the authors feeling that explanation and learning, required from UFIMA, imply using corresponding Artificial Intelligence techniques. Explanation, learning, pro-activity, autonomy, cooperation, delegation and negotiation, lead to implement the architecture model we have proposed in this article as a multi intelligent agent architecture.

The management model this article proposed is a general one because of the generality of the adopted architecture model. We presented the possible implementation options that should be decided, when implementing the solution, taking into consideration the available services, the user device capabilities and operator policies. In future work, we will describe an ongoing implementation of this model in a SIP framework.

\section{References}

1. Cameron, E.J. et al: A feature interaction benchmark for IN and beyond. Feature Interactions in Telecommunications Systems. IOS Press, Amsterdam (1994) 1-23

2. Amer, M. et al: Feature interactions resolution using fuzzy policies. Feature Interactions in Telecommunications Systems. IOS Press, Amsterdam (2000) 94-112

3. Keck, D.O., Kuehn, P.J.: The feature and service interaction problem in telecommunications systems: a survey. IEEE Transactions on Software Engineering, Vol. 24. New York (1998) 779-796

4. Chentouf, Z., Khoumsi, A., Cherkaoui, S.: Conceptual foundations of user preference modeling. International Conference on Network Control and Engineering (NETCON'2003). Kluwer Academic Publishers, New York (2003)

5. Chentouf, Z., Cherkaoui, S., Khoumsi, A.: Implementing online Feature Interaction detection in SIP environment: early results. $10^{\text {th }}$ International Conference on Telecommunications (ICT'2003), Tahiti (2003)

6. Rosenberg, J. et al : SIP: Session Initiation Protocol. RFC 3261. IETF (2002)

7. JAIN. http://java.sun.com/products/jain

8. 3GPP. http://www.3gpp.org

9. Lennox, J., Schulzrinne, H.: CPL: a language for user control of Internet telephony services. IETF Draft (2000)

10. Chentouf, Z., Khoumsi, A., Cherkaoui, S.: Détection hors-ligne d'interactions de services dans les réseaux hétérogènes de télécommunications. Colloque Francophone sur l'ingénierie des Protocoles (CFIP'2002). Hermes, Paris (2002)

11. Griffeth, N.D., Velthuijsen, H.: The negotiating agents approach to runtime interaction resolution. Feature Interactions in Telecommunications Systems. IOS Press, Amsterdam (1994) 217-235

12. Blom, J. et al: Automatic detection of feature interactions in temporal logic. Feature Interactions in Telecommunications Systems. IOS Press, Amsterdam (1995) 1-19 
13. Gammelgaard, A., Kristensen, J.E.: Interaction detection, a logical approach. Feature Interactions in Telecommunications Systems. IOS Press, Amsterdam (1994) 178-196

14. Nakumara, M. et al: Petri-net based detection method for non-deterministic feature interactions and its experimental evaluation. Feature Interactions in Telecommunications Systems. IOS Press, Amsterdam (1997) 138-152

15. Gibson, P., Mery, D.: Telephone feature verification: translating SDL to TLA+. Eighth SDL Forum (SDL'1997), Evry, France (1997)

16. Amyot, D. et al: Feature description and Feature Interaction analysis with Use Case Maps and LOTOS. Feature Interactions in Telecommunications Systems. IOS Press, Amsterdam (2000)

17. Charnois, T.: A natural language processing approach for avoidance of feature interactions. Feature Interactions in Telecommunications Systems. IOS Press, Amsterdam (1997) 347363

18. Cherkaoui, S., Khoumsi, A.: Mobile and static agents for service interactions resolution in telecommunication environments. 9th IEEE International Conference on Telecommunications (ICT'2002), Beijing (2002)

19. Khoumsi, A.: Detection and resolution of interactions between services of telephone networks. Feature Interactions in Telecommunications Systems. IOS Press, Amsterdam (1997) 78-92

20. Blom, J., Johnsson, B., Kempe, L.: Using temporal logic for modular specification of telephone services. Feature Interactions in Telecommunications Systems. IOS Press, Amsterdam (1994) 197-216

21. Tsang, S., Magill, E.H.: Behavior based run-time feature interaction detection and resolution approaches for intelligent networks. Feature Interactions in Telecommunications Systems. IOS Press, Amsterdam (1997) 254-270

22. Rizzo, M., Garyfalos, A.: Using SIP to negotiate over user requirements in personalized Internet Telephony services. SIP'2000, Paris (2000)

23. Kolberg, M., Magill, E.H.: Handling incompatibilties between services deployed on IPbased networks. IEEE Intelligent Networks (IN'2001). IEEE Press, Boston (2001)

24. Jennings, N., Sycara, N., Wooldridge, M.: A roadmap of agent research and development. International Journal of Autonomous Agents and Multi-Agent Systems, Vol. 1 (1998) 7 38 